\title{
Using Baidu Search Index to monitor and predict AIDS, syphilis and gonorrhea in China
}

1: School of Public Health, Sun Yat-sen University 2: School of Public Health (Shenzhen), Sun Yat-sen University 3: University of North Carolina Project China 4: Central Clinical School, Monash University

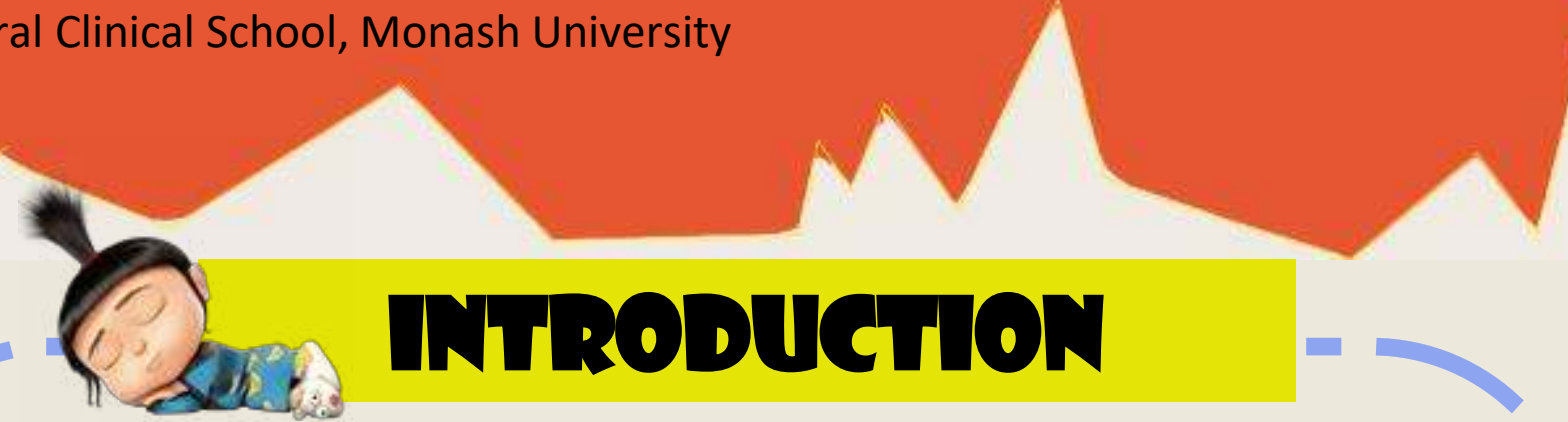

- AIDS, syphilis and gonorrhea are prevalent STDs in China listed as category B notifiable infectious diseases in the law.

- Category B notifiable infectious diseases are required to be reported within 24 hours in the national surveillance system, however there is a 1-2 week delay of publication of official statistics. Using search data to predict can potentially overcome publication delay and help health authorities to make preemptive preventative and treatment plans.

- In China there has been no research evaluating the use of search engine data in predicting AIDS and other STDs. Baidu holds the highest search engine market penetration rate in China (93.1\% in December, 2015). It is currently the most representative tool to measure search engine users' behaviors in the country.

Keywords Selection and Filtering

\section{Search Index Composition}

$$
\text { A }
$$
about celebrities or media hype.

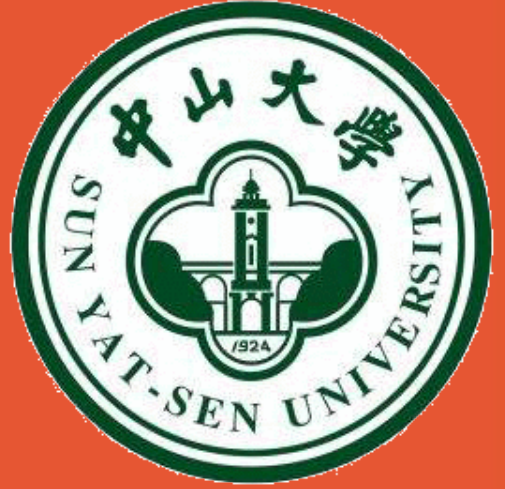

Notified incidence case counts:

\section{Official website of National Health and} Family Planning Commission

The search volume data of keywords: http://index.baidu.com/

The original keywords set: http://tool.chinaz.com/

\section{Exclusion Criteria:}

1.Words irrelevant to the incidence of AIDS, syphilis and gonorrhea, such as 'The World AIDS Day' etc.

2. Words with an interrupted time series representing Baidu search index, i.e. anecdotes

3. Words that few people use in real life search whose Baidu search index was always zero in a long period of time (6 months or longer).

4. Words whose Baidu search index's

Spearman's rank correlation coefficients with the monthly reported incidence case counts was less than 0.4

$$
\text { Weight }_{i}=\frac{\rho_{i}}{\sum_{i=1}^{n} \rho_{i}}
$$

\section{Composite Search Index \\ $=\sum_{i=1}^{n}$ Weight $_{i} *$ Keyword $_{i}$}

$\rho_{i}$ : spearman's rank correlation coefficient of the $i^{\text {th }}$ word Keyword $_{i} \&$ Weight $_{i}$ : the Baidu search index of $i^{\text {th }}$ word and the weight of it

$$
\begin{aligned}
& \text { oCC } \boldsymbol{C}_{\boldsymbol{t}}=\beta_{0} * \text { OCC }_{\boldsymbol{t}_{-1}}+\beta_{1} * \text { indexnt }+\beta_{2} * \\
& \text { index }_{n\left(t_{-} 1\right)}+\varepsilon
\end{aligned}
$$

- $O C C_{t} \& O C C_{t-1}$ : the notified incidence case counts in $t^{t h}$ month and in $(t-1)^{t h}$ month

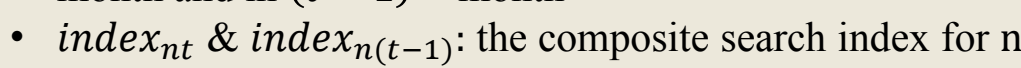
keywords in $t^{\text {th }}$ month and in $(t-1)^{t h}$ month

- $\beta_{0}, \beta_{1}, \beta_{2} \& \varepsilon$ : the coefficients and the error term

\section{MLTIOOS}

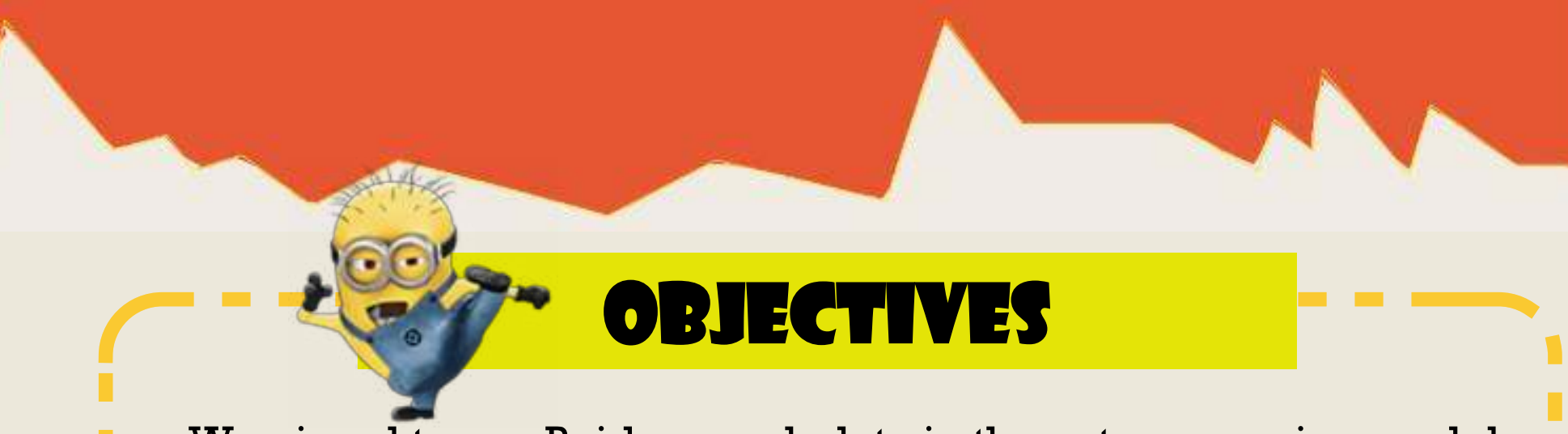

We aimed to use Baidu search data in the autoregressive model combined with past notified incidence case counts to monitor and predict AIDS, syphilis and gonorrhea in China.

\section{RESULTS}

Following the keywords exclusion criteria, 21 keywords for AIDS, 12 for syphilis and 3 for gonorrhea remained to construct the composite search index.
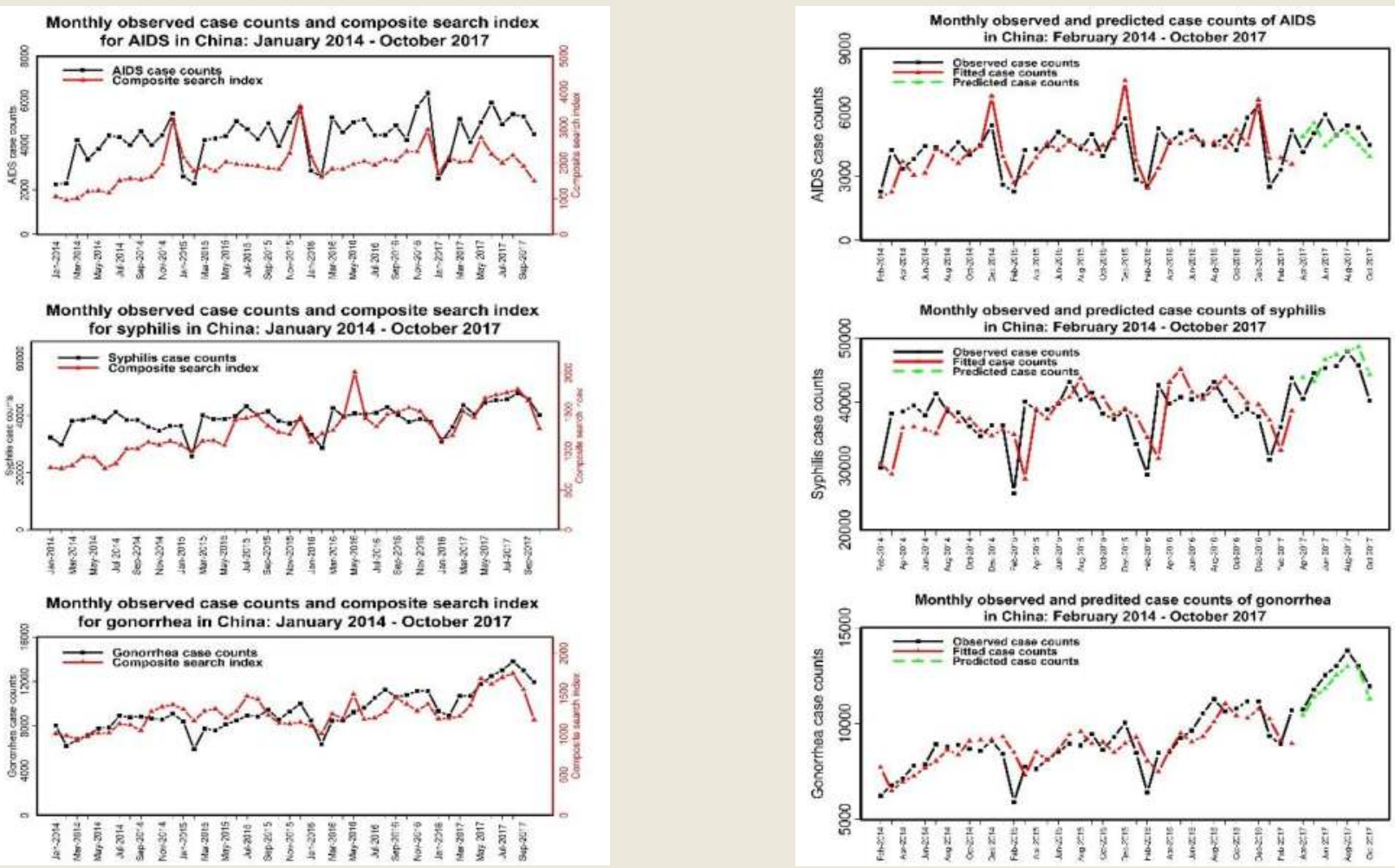

Figure 1. Trends of incidence case Figure 2. Observed case counts counts and composite search index and prediction based on the model Table 1. Keywords list included Table 2. Statistical results of mode in the composite search index for AIDS, syphilis and gonorrhea
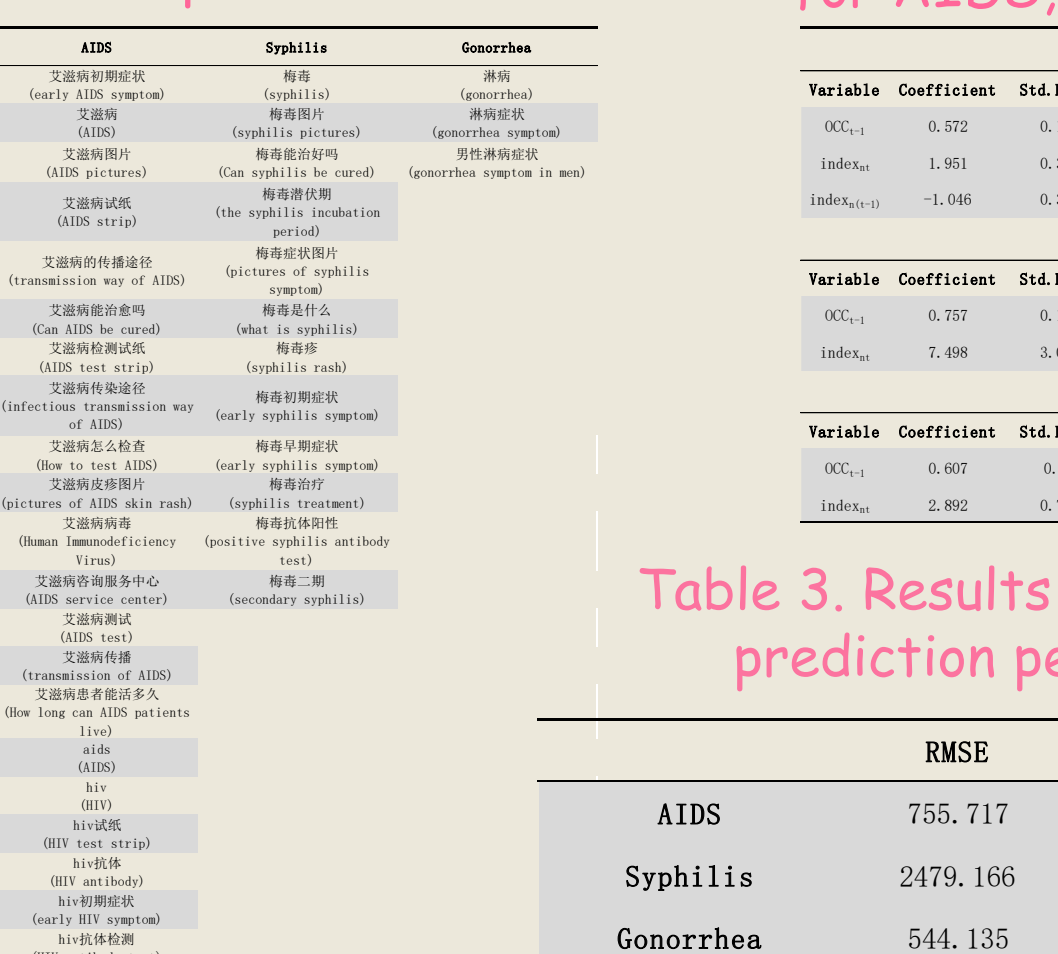